\title{
Forming Jupiter, Saturn, Uranus and Neptune in Few Million Years by Core Accretion
}

\author{
Omar G. Benvenuto ${ }^{1,2,3}$, Andrea Fortier ${ }^{1,3,4}$, Adrián Brunini ${ }^{1,3,5}$ \\ 1.- Facultad de Ciencias Astronómicas y Geofísicas, Universidad Nacional de La Plata (UNLP), \\ 1900 La Plata, Argentina. \\ 2.- Member of the Carrera del Investigador Científico, Comisión de Investigaciones Científicas de \\ la Provincia de Buenos Aires (CIC), Argentina. \\ 3.- Instituto de Astrofísica de La Plata (IALP), CCT-Consejo Nacional de Investigaciones Científicas \\ y Técnicas (CONICET), UNLP, Argentina. \\ 4.- Fellow of CONICET. \\ 5.- Member of the Carrera del Investigador Científico, CONICET.
}

\begin{abstract}
Giant planet formation process is still not completely understood. The current most accepted paradigm, the core instability model, explains several observed properties of the solar system's giant planets but, to date, has faced difficulties to account for a formation time shorter than the observational estimates of protoplanetary disks' lifetimes, especially for the cases of Uranus and Neptune. In the context of this model, and considering a recently proposed primordial solar system orbital structure, we performed numerical calculations of giant planet formation. Our results show that if accreted planetesimals follow a size distribution in which most of the mass lies in 30-100 meter sized bodies, Jupiter, Saturn, Uranus and Neptune may have formed according to the nucleated instability scenario. The formation of each planet occurs within the time constraints and they end up with core masses in good agreement with present estimations.
\end{abstract}

key words: Planetary formation; Accretion; Planetesimals.

Correspondence should be directed to: Omar G. Benvenuto, Facultad de Ciencias Astronómicas y Geofísicas de La Plata, Paseo del Bosque S/N (1900) La Plata, Argentina. E-mail: obenvenu@fcaglp.fcaglp.unlp.edu.ar 
Terrestrial planets are widely believed to form in the inner solar nebula through the accretion of a population of centimeter to kilometer sized planetesimals orbiting the early Sun (Wetherill 1990). The core-accretion model states that this process also occurred in the outer solar nebula (Mizuno 1980; Pollack et al. 1996). When a solid embryo has grown to few thousandths of the Earth mass, a gaseous envelope in hydrostatic equilibrium begins to bind to it. This equilibrium is achieved by the balance between the heating due to the energy released by the incoming planetesimals and the gravity of the core. As the core grows and planetesimals exhaust, the gaseous envelope eventually can no longer be supported and contracts. This leads to the last stage of giant planet formation, a short period during which the protoplanet quickly gains the bulk of its total mass (Pollack et al. 1996). On the other hand, the first stage of the process is dominated by the formation of the core. The time scale of core growth is strongly dependent on several factors, the initial disk surface density of solids in the giant planet region, $\Sigma$, and the radius of accreted planetesimals being among the most important ones.

The first calculations that self-consistently considered both the accretion of gas as well as planetesimals showed that, even prescribing an unrealistically fast core accretion rate, with a surface density of solids of $\Sigma=10 \mathrm{~g} \mathrm{~cm}^{-2}$ at $5.2 \mathrm{AU}$, Jupiter takes 6 million years (My) to complete its formation (Pollack et al. 1996, updated by Hubickyj et al. 2005)1, and Saturn more than 10 My (Pollack et al. 1996). However, according to observations, the lifetime of circumstellar disks at the Jupiter-Saturn region is between 3-8 My, while beyond 10 AU they are expected to last somewhat longer (Hillenbrand 2005). This fact limits the formation time of gaseous planets. Increasing the local density speeds up the formation process, but at the cost of building too massive cores: interior models of Jupiter that agree with all observational constraints show that its core mass is at most $12 \mathrm{M}_{\oplus}$ (Guillot 2005), whereas more recent calculations give core masses of 14 - $18 \mathrm{M}_{\oplus}$ (Militzer et al. 2008). In the case of Saturn, its core mass is between 9-22 $\mathrm{M}_{\oplus}$ (Guillot 2005). Recent improved core accretion calculations adopt more adequate solid accretion rates for the cores (Fortier et al. 2007, 2009), or take into account local density patterns in the disk (Klahr \& Bodenheimer 2006), but nevertheless they have not solved the time scale problem. Other recent models showed that the time scale for envelope growth depends on the assumed dust grain opacity (Ikoma et al. 2000). Grains entering the protoplanetary envelope may coagulate and settle down quickly. As a consequence, the correct opacity may be far lower than usually believed (Podolak 2003), easing an early envelope contraction that allows the formation of Jupiter in less than 3 My (Hubickyj et al. 2005). However, the time scale to form the core, one of the main problems of the core accretion model, is not substantially modified and, to date, no self-consistent calculations of the opacity in the protoplanet's atmosphere have been performed (Movshovitz \&

\footnotetext{
${ }^{1}$ This result corresponds to the simulation $10 \mathrm{H}^{\infty}$ of Hubickyj et al. (2005) computed with full interstellar opacities.
} 
Podolak 2008). Improved solid surface density calculations (Dodson - Robinson et al. 2008) allow to compute formation models of Saturn consistent with circumstellar disk lifetimes. However, their simulations end with a total mass of heavy elements higher than current estimations for Saturn. In all core accretion calculations performed so far, a single sized planetesimal population has been assumed. However, remarkably, the accretion rate of solids is strongly dependent on the planetesimal size. Therefore, self-consistent numerical simulations, in which the core is formed through accretion of planetesimals following a size distribution are in order. At early stages, larger planetesimals grow faster than smaller ones, resulting in a runaway growth of the largest (Greenberg et al. 1978; Wetherill \& Stewart 1989; Kokubo \& Ida 1996). Runaway bodies then become protoplanetary embryos that grow by the accretion of small planetesimals. When the mass of a protoplanet exceeds a critical value far lower than the Earth mass, runaway growth slows down (Ida \& Makino 1993) and switches to oligarchic growth (Kokubo \& Ida 1998), in which similar-sized protoplanets dominate the planetesimal system. During the oligarchic regime only protoplanets grow as there is no substantial accretion among planetesimals. Remarkably, solid bodies in the swarm are not of uniform size but span over a wide interval ranging from millimeter to kilometer.

Numerical simulations show that the mass distribution of these residual planetesimals may be represented by a single or piecewise power law $d n_{c}(m) / d m \propto n(m) \propto m^{-\alpha}$, where $n_{c}(m)$ is the number of bodies larger than a given mass $m$ and $n(m)$ is the number of bodies in a linear mass bin. For a constant value of $\alpha$ the mass in the interval is $\int n m d m \propto m^{2-\alpha}$. Depending on the value of $\alpha$ the mass in the interval would be mostly contained in small $(\alpha>2)$ or large $(\alpha<2)$ planetesimals. Wetherill and Stewart (1993) studied the evolution of the planetesimal system considering an initial population of planetesimals whose radius is $\sim 10 \mathrm{~km}$ that evolved only through collisions and fragmentation. They found that planetesimal size distribution relaxes to a piecewise power law: a population of small planetesimals due to fragmentation $(\alpha \sim 1.7)$ and a population of large planetesimals that follow an accretive regime $(\alpha \sim 2.5)$ which, in turn, contained most of the mass of the system. Kokubo and Ida (2000) studied through N-body simulations the evolution of planetesimal size and they found that, in the oligarchic regime, large planetesimals follow a continuous power law distribution with $2<\alpha<3$. However, these studies do not take into account the effects due to magneto-rotational instability turbulence, which establish the predominance of an erosive rather than an accretive regime for planetesimal growth, nor the existence of dead zones that could favor accretion among planetesimals (Ida et al. 2008). On the other hand, recent laboratory experiments show that reaccumulation of fragmentation debris can lead to the formation of planetesimals (Teiser and Wurm, 2009). These effects could have a substantial impact on the accretion process of planetesimals, specially in the small planetesimal tail of the distribution. Evidently, the primordial planetesimal size distribution in the protoplanetary 
disk is still an open problem. In our study, for the sake of simplicity we shall consider a continuous power law distribution, adopting for the exponent the value $\alpha=2.5$. In view of the present status of the theory of planetesimal growth, we consider plausible such a distribution.

Another important point to take into account is that the primordial configuration of the outer solar system was probably not the one we know today. Numerical simulations (Tsiganis et al. 2005) assuming an originally compact planetary system (the Nice model) were able to reproduce the main observational constraints regarding the present orbital structure of the outer solar system, that was achieved after the migration of the fully formed giant planets to their present location. In $\sim 50 \%$ of these simulations Uranus and Neptune switch places, explaining naturally why Neptune's mass is larger than that of Uranus. The Nice model implies a solid surface density profile $\Sigma \propto a^{-2.168}$, where $a$ is the distance from the Sun (Desch 2007).

In our model, in order to be consistent with the Nice model, we placed Jupiter, Saturn, Uranus and Neptune's embryos at $a=5.5,8.3,14$, and 11 AU respectively. We calculate the in situ formation of these planets. This assumption, although a simplification of the model, is compatible with recent studies of the formation of planetary systems. Thommes et al. (2008) found that solar system analogs come out if the gas giants do not undergo significant migration and remain in nearly circular orbits. In all our simulations, the initial core and gaseous envelope masses were set to $\sim 10^{-2} \mathrm{M}_{\oplus}$ and $\sim 10^{-10} \mathrm{M}_{\oplus}$ respectively. The equations governing the evolution of the gaseous envelope were solved coupled self-consistently to the planetesimal accretion rate, employing a standard finite difference (Henyey) method and detailed constitutive physics as described in our previous work (Fortier et al. 2007, 2009). We adopted the equation of state of Saumon et al. (1995), the grain opacities of Pollack et al. (1985) and, for temperatures above $10^{3} \mathrm{~K}$, the molecular opacities of Alexander \& Ferguson (1994).

The accretion of planetesimals modify the solid surface density around the planet which, together with their relative velocities, control the solids accretion rate. The relative velocities are calculated assuming the equilibrium between the gravitational perturbations due to the embryo and the dissipation due to gas drag. Since the oligarchic regime dominates the time scale for the formation of the core, we prescribed this accretion rate during the whole simulation (Fortier et al. 2007, 2009). To approximately mimic the effects due to neighboring planets (Hubickyj et al. 2005), after the planet reaches the first maximum in the solid accretion rate, we inhibited a further growth of the local planetesimal surface density, i.e., we no longer allow planetesimals to refill the planetary feeding zone. We assumed that all captured planetesimals sink onto the solid core and that their mass density is equal to $1.5 \mathrm{~g} \mathrm{~cm}^{-3}$. We approached the continuous planetesimal size distribution with a discrete one of nine sizes from $30 \mathrm{~m}$ to $100 \mathrm{~km}$ of radius, geometrically evenly spaced. Considering a non homogeneous population is very important as planetesimal dynamics is affected by nebular drag. Small planetesimals suffer a stronger damping of random velocities, 
increasing this way the accretion rate (Safronov 1969); but they are also subject to faster orbital decay, reducing the planetesimal surface density in the neighborhood of the protoplanet. In the nebular model we are using, the accretion time scale of planetesimals whose radius is $r \geq 30 \mathrm{~m}$ is shorter than the corresponding time scale of orbital decay (Chambers 2006; Brunini \& Benvenuto 2008). This situation reverts at $r \sim 20 \mathrm{~m}$. Therefore, the giant planets of the solar system should have formed essentially through accretion of planetesimals larger than $r \sim 30 \mathrm{~m}$, as smaller planetesimals could not have been efficiently accreted. It is worth mentioning that a nebular model based on the present planetary masses, like the minimum mass solar nebula, does not account for the primordial solid mass comprised in the planetesimals that were not accreted by the planets. Then, the estimations of the nebular surface density adopted in this article only take into account the mass contained in planetesimals that have chances to be accreted.

Fig. 1 shows the results for the formation of Jupiter. Allowing for a distribution of planetesimal sizes where the most abundant ones are those of $r=30-80 \mathrm{~m}$, Jupiter grows much faster than considering a typical single size population (e.g. $r=10 \mathrm{~km}$ ). Note that the model corresponding to $\Sigma=11 \mathrm{~g} \mathrm{~cm}^{-2}$ forms timely and achieves a core mass compatible with currently accepted estimations. The key ingredient that speeds up planetary growth is the dependence of the crosssection of the planet as a function of the planetesimal size. This, in turn, is determined by the drag force suffered by planetesimals after entering the planetary envelope, which is proportional to the reciprocal of the planetesimal radius (Podolak et al. 1988). If there was no gas bound to the planet, the core would grow only by inelastic collisions with planetesimals. For this process, the cross-section is given by the projected area of the planet enhanced by gravitational focusing. Gas drag modifies planetesimal trajectories, eventually trapping them in the planet, greatly enlarging the cross-section (Pollack et al. 1996; Fortier et al. 2007, 2009).

Let us consider the conditions for the formation of the giant planets beyond Jupiter's orbit. Regarding Uranus and Neptune, models consistent with available observations predict a gaseous mass fraction far lower than those of Jupiter and Saturn. The upper limits for the gas content of Uranus and Neptune are of $5.0 \mathrm{M}_{\oplus}$ and $4.7 \mathrm{M}_{\oplus}$ respectively (Podolak et al. 2000), while the absolute lower limit for both of them is of $0.5 \mathrm{M}_{\oplus}$ (Guillot 2005).

Fig. 2 shows the results corresponding to the four giant planets of the solar system computed separately, together with plausible values for their core masses (Guillot 2005; Podolak et al. 2000). Here the four giant planets are formed within the estimated lifetime of protoplanetary disks. Other simulations (Alibert et al. 2005) were able to form Jupiter and Saturn in less than 2.5 My but with Saturn undergoing a migration in conflict with the Nice model (Tsiganis et al. 2005) and neglecting the oligarchic regime for the growth of the core. On the other hand, Desch (2007) estimated the growth time for the cores of the four giant planets in the framework of the Nice model. Although he did not consider the presence of the planets' atmosphere, his results support 
the possibility of the formation of all the giant planets of the solar system in less than $10 \mathrm{My}$, provided that small planetesimals were the building blocks of the cores.

Regarding the mass of the core, as can be seen from Fig. 2, Uranus and Saturn's fall inside the error bar while Jupiter is compatible with the top of its error bar. Neptune's model presents a core smaller than current estimations. Nevertheless, we should remark that Neptune's observations are by far the more inaccurate ones (Podolak et al. 2000).

If we consider that the size distribution of planetesimals is represented by 7 species where the minimum radius is $r=100 \mathrm{~m}$, instead of 9 species starting with $r=30 \mathrm{~m}$, the formation time of the four giant planets is lengthened, being the longest one that of Uranus ( $\sim 13 \mathrm{My}$ ). Moreover, if we consider that most of the planetesimal mass lies in kilometer-sized bodies, the formation time of the ice giants results much longer than the estimated lifetime of the protoplanetary disk.

In order to evaluate the effects of the above mentioned inhibition on the solids accretion rate, we also computed the growth of the four giant planets without such effect. The results are presented in Fig. 3. In this case, planetary cores are more massive: Jupiter, Saturn, Neptune and Uranus form cores of 20,19,13 and $11 \mathrm{M}_{\oplus}$ respectively. The only core mass outside, but near its error bar is that of Jupiter. However, in view of the disagreement between different research groups on the actual value of Jupiter's core mass, we judge our result as acceptable. Also, note that imposing no inhibition makes the whole planetary formation process even somewhat faster.

In this study we performed calculations of giant planet formation in the framework of the nucleated instability model, taking into account the oligarchic regime for the growth of the core and a primordial configuration of the solar system according to the Nice model. Also, we considered a size distribution for the accreted planetesimals in which most of the mass lies in the smallest planetesimals of the population. This study shows that the formation of the four giant planets of the solar system is compatible with the observational constraints imposed by dust disk lifetimes. We found that to account for the formation of Jupiter, Saturn, Uranus and Neptune in less than 10 My most of the mass of accreted planetesimals has to be in planetesimals whose radius is $\lesssim 100 \mathrm{~m}$. We should remark that if we assume a power law with $\alpha<2$, planetary embryos would grow on a too long timescale. In this sense, we have chosen particularly favorable conditions for the occurrence of planetary formation within the currently accepted constraints (timescales and core masses). Hopefully, future investigation on the theory of planetesimal formation will provide further insights on the primordial size distribution of planetesimals in the protoplanetary disk.

\section{Acknowledgments}

The authors thank R.E. Martinez and R.H. Viturro for the technical support.

\section{References}


Alexander, D. R., Ferguson, J. W. 1994. Low-temperature Rosseland opacities. Astrophysical Journal 437, 879-891.

Alibert, Y., Mousis, O., Mordasini, C., Benz, W. 2005. New jupiter and saturn formation models meet observations. Astrophysical Journal 626, L57-L60.

Brunini, A., Benvenuto, O. G. 2008. On oligarchic growth of planets in protoplanetary disks. Icarus 194, 800-810.

Chambers, J. 2006. A semi-analytic model for oligarchic growth. Icarus 180, 496-513.

Desch, S. J. 2007. Mass distribution and planet formation in the solar nebula. Astrophysical Journal $671,878-893$.

Dodson-Robinson, S. E., Bodenheimer, P., Laughlin, G., Willacy, K., Turner, N. J., Beichman, C. A. 2008. Saturn Forms by Core Accretion in 3.4 Myr. Astrophysical Journal Letters, 688, L99 Fortier, A., Benvenuto, O. G., Brunini, A. 2007. Oligarchic planetesimal accretion and giant planet formation. Astronomy and Astrophysics 473, 311-322.

Fortier, A., Benvenuto, O. G., Brunini, A. 2009. Oligarchic planetesimal accretion and giant planet formation II. Astronomy and Astrophysics, in press (DOI: 10.1051/0004-6361/200811367).

Greenberg, R., Hartmann, W. K., Chapman, C. R., Wacker, J. F. 1978. Planetesimals to planets - Numerical simulation of collisional evolution. Icarus 35, 1-26.

Guillot, T. 2005. The interiors of giant planets: Models and outstanding questions. Annual Review of Earth and Planetary Sciences 33, 493-530.

Hillenbrand, L. A. 2005. Observational constraints on dust disk lifetimes: implications for planet formation. ArXiv Astrophysics e-prints arXiv:astro-ph/0511083.

Hubickyj, O., Bodenheimer, P., Lissauer, J. J. 2005. Accretion of the gaseous envelope of Jupiter around a 5 - 10 Earth-mass core. Icarus 179, 415-431.

Ida, S., Makino, J. 1993. Scattering of planetesimals by a protoplanet - Slowing down of runaway growth. Icarus 106, 210-227.

Ida, S., Guillot, T., Morbidelli, A. 2008. Accretion and Destruction of Planetesimals in Turbulent Disks. Astrophysical Journal 686, 1292-1301.

Ikoma, M., Nakazawa, K., Emori, H. 2000. Formation of giant planets: dependences on core accretion rate and grain opacity. Astrophysical Journal 537, 1013-1025.

Klahr, H., Bodenheimer, P. 2006. Formation of giant planets by concurrent accretion of solids and gas inside an anticyclonic vortex. Astrophysical Journal 639, 432-440.

Kokubo, E., Ida, S. 1996. On runaway growth of planetesimals. Icarus 123, 180-191.

Kokubo, E., Ida, S. 1998. Oligarchic growth of protoplanets. Icarus 131, 171-178.

Kokubo, E., Ida, S. 2000. Formation of protoplanets from planetesimals in the solar nebula. Icarus $143,15-27$. 
Militzer, B., Hubbard, W. B., Vorberger, J., Tamblyn, I., Bonev, S. A. 2008, Astrophysical Journal Letters, 688, L45

Mizuno, H. 1980. Formation of the giant planets. Progress of Theoretical Physics 64, 544-557.

Movshovitz, N., Podolak, M. 2008. The opacity of grains in protoplanetary atmospheres. Icarus 194, 368-378.

Podolak, M. 2003. The contribution of small grains to the opacity of protoplanetary atmospheres. Icarus 165, 428-437.

Podolak, M., Pollack, J. B., Reynolds, R. T. 1988. Interactions of planetesimals with protoplanetary atmospheres. Icarus 73, 163-179.

Podolak, M., Podolak, J. I., Marley, M. S. 2000. Further investigations of random models of Uranus and Neptune. Planetary and Space Science 48, 143-151.

Pollack, J. B., McKay, C. P., Christofferson, B. M. 1985. A calculation of the Rosseland mean opacity of dust grains in primordial solar system nebulae. Icarus 64, 471-492.

Pollack, J. B., Hubickyj, O., Bodenheimer, P., Lissauer, J. J., Podolak, M., Greenzweig, Y. 1996. Formation of the giant planets by concurrent accretion of solids and gas. Icarus 124, 62-85.

Safronov, V.S. 1969. Evolution of of the protoplanetary cloud and formation of the earth and planets. Nauka, Moscow.

Saumon, D., Chabrier, G., van Horn, H. M. 1995. An Equation of State for Low-Mass Stars and Giant Planets. Astrophysical Journal Supplement Series 99, 713.

Teiser, J., Wurm, G. 2009. High-velocity dust collisions: forming planetesimals in a fragmentation cascade with final accretion. Monthly Notices of the Royal Astronomical Society 393, 1584-1594. Thommes, E. W., Matsumura, S., Rasio, F. A. 2008. Gas disks to gas giants: Simulating the birth of planetary systems. Science 321, 814-817.

Tsiganis, K., Gomes, R., Morbidelli, A., Levison, H. F. 2005. Origin of the orbital architecture of the giant planets of the Solar System. Nature 435, 459-461.

Wetherill, G. W. 1990. Formation of the earth. Annual Review of Earth and Planetary Sciences 18, 205-256.

Wetherill, G. W., Stewart, G. R. 1989. Accumulation of a swarm of small planetesimals. Icarus $77,330-357$.

Wetherill, G. W., Stewart, G. R. 1993. Formation of planetary embryos - Effects of fragmentation, low relative velocity, and independent variation of eccentricity and inclination Icarus 106, 190-209. 


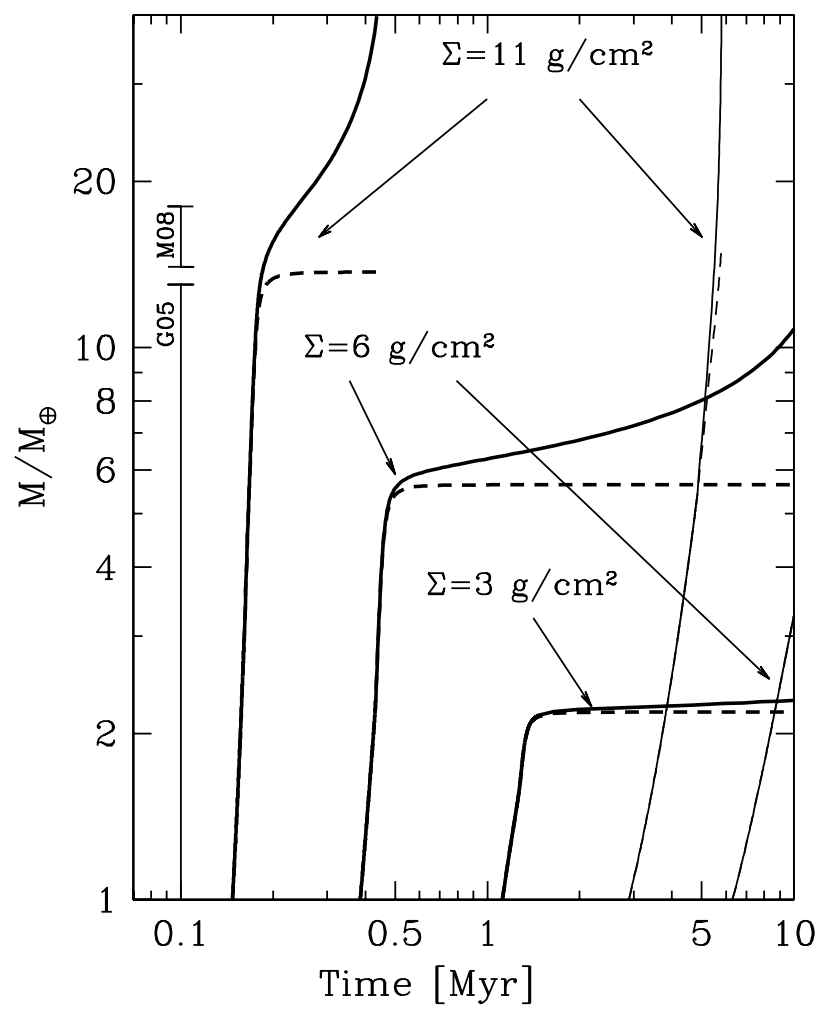

Figure 1: The growth of Jupiter for different surface densities. Thick solid (dashed) lines depict the growth of the total (core) mass for models computed with a size distribution for the accreted planetesimals. Thin lines stand for the case of a single sized population of planetesimals with $r=10 \mathrm{~km}$. The error bars depict the currently accepted values for Jupiter's core mass. G05 denotes the values given by Guillot (2005) while M08 stands for the results presented by Militzer et al. (2008). $\Sigma$ represents the assumed values for the initial disk surface density of solids in the Jupiter region. Note that the formation is a two step process. First, the core grows by planetesimal accretion, whose gravitational energy release inhibits the gaseous envelope from undergoing a premature contraction. When the neighborhood of the protoplanet is depleted from planetesimals, the core mass gets its asymptotic value and a runaway gas accretion is established. Quickly, the planet reaches its final mass $\left(318 \mathrm{M}_{\oplus}\right)$. Clearly, the considered size distribution of planetesimals yields a formation time scale much shorter than considering a single planetesimal size of $r=10 \mathrm{~km}$. In particular, with a local surface density of $11 \mathrm{~g} \mathrm{~cm}^{-2}$, we obtain an acceptable core mass value and a comfortably short formation time. Therefore we assume that this result scales the density throughout the entire protoplanetary disk. 


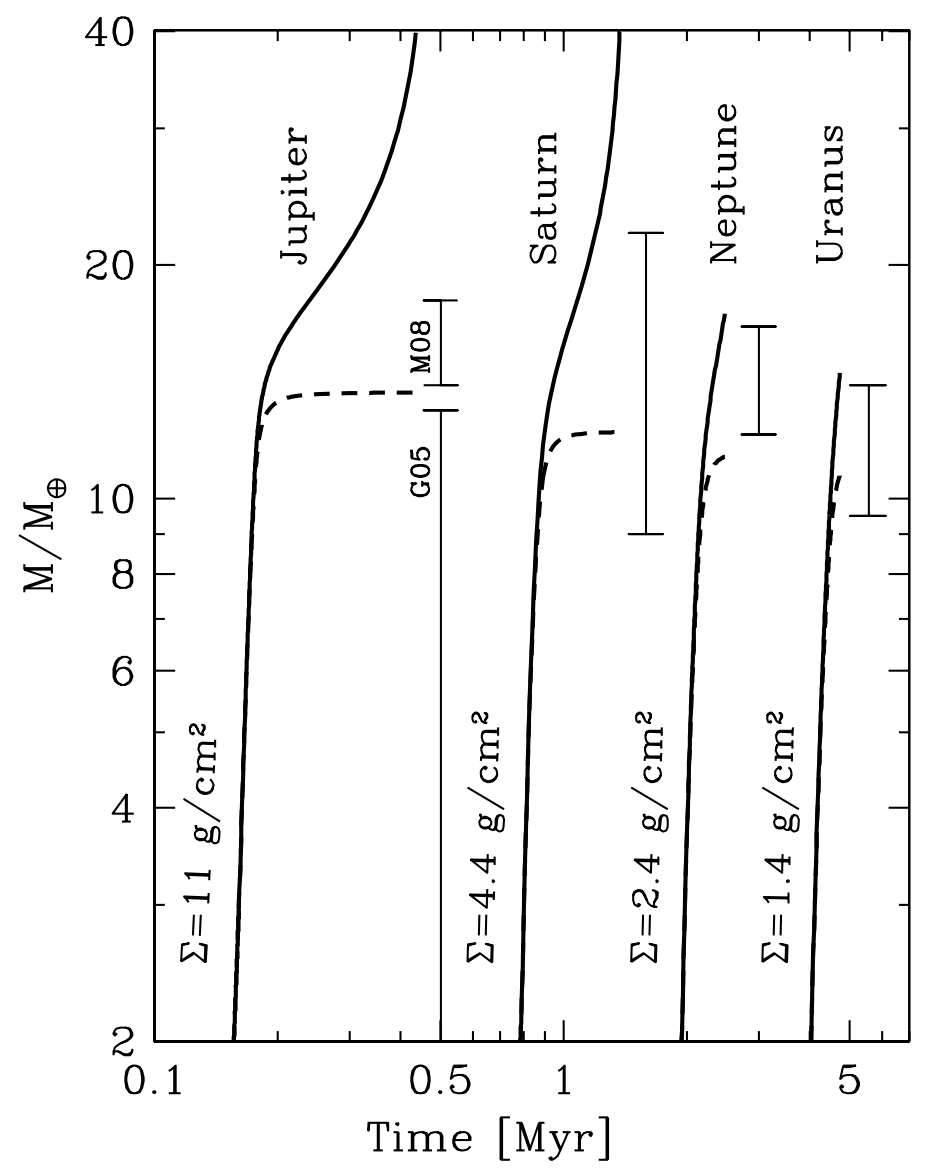

Figure 2: The growth of the four giant planets. Solid (dashed) lines depict the growth of the total (core) mass for models computed with a size distribution for the accreted planetesimal. The values of $\Sigma$ correspond to the initial surface density of solids at the position of each embryo. The error bars depict the currently accepted values for the core masses of each planet. For the case of Jupiter, as in Fig. 1, the lower error bar is that of Guillot (2005), whereas the upper one corresponds to the results given by Militzer et al. (2008). Simulations were stopped when the final mass of each planet was reached (approximately 318, 95, 14, and $17 \mathrm{M}_{\oplus}$ for Jupiter, Saturn, Uranus and Neptune respectively). The formation times are: Jupiter 0.44 My, Saturn $1.4 \mathrm{My}$, Neptune 2.5 My and Uranus 4.75 My. 


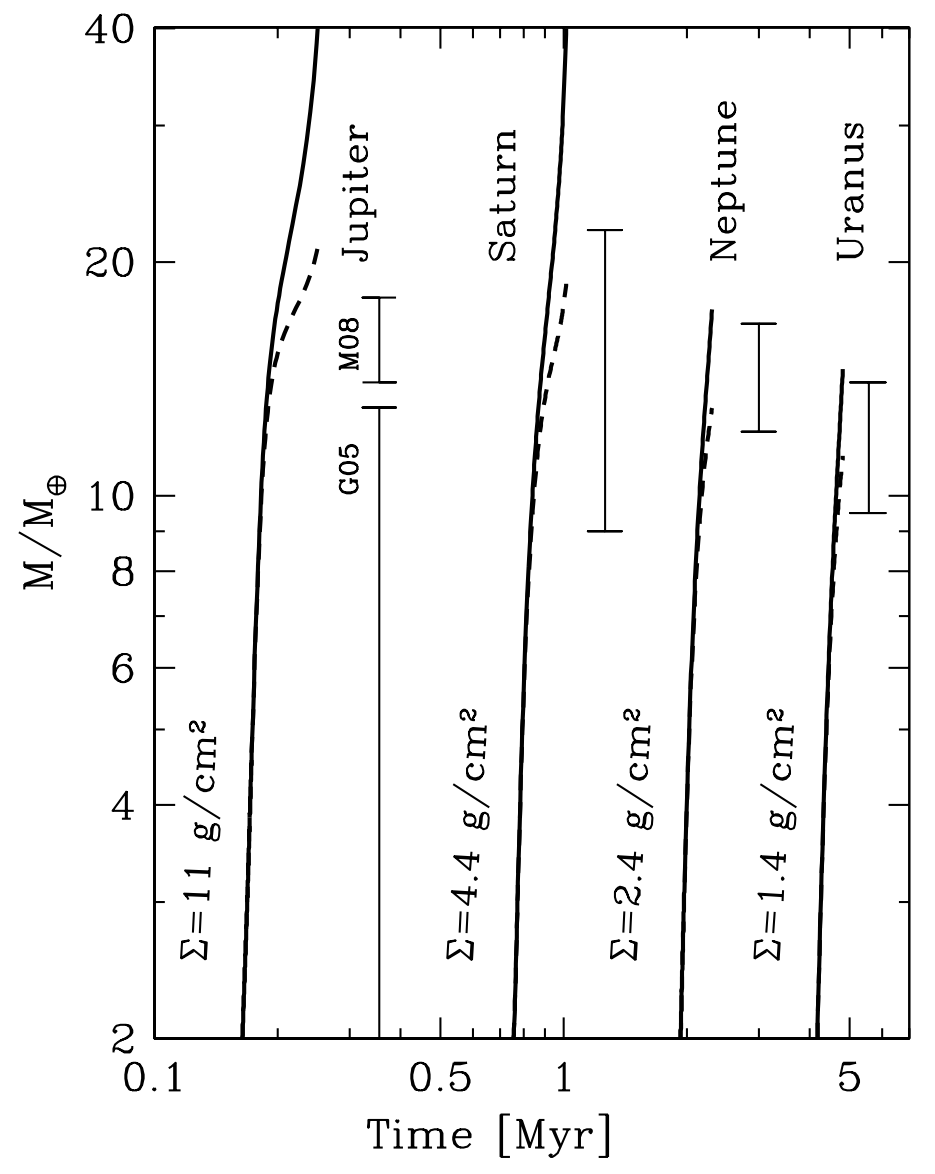

Figure 3: Same as Fig. 2 but without imposing any inhibition on the solid mass accretion rate. 